\title{
Downlink Overloaded Multiple Access Based on Constellation Expansion
}

\author{
Alberto G. Perotti*, Jaap van de Beek ${ }^{\dagger *}$ and Branislav M. Popović* \\ * Huawei Technologies Sweden AB \\ Skalholtsgatan 9-11, SE-16494 Kista, Sweden \\ E-mails: \{alberto.perotti, branislav.popovic\} \\ @huawei.com \\ $\dagger$ Luleå University of Technology \\ Dept. of Computer Science, Electrical and Space Engineering \\ SE-971 87 Luleå, Sweden \\ E-mail: jaap.vandebeek@ltu.se
}

\begin{abstract}
A new overloaded multiple access scheme for the downlink of wireless systems is presented. It performs simultaneous transmission of multiple data streams intended for users experiencing similar signal-to-noise ratios (SNR). The proposed scheme performs stream multiplexing by mapping the coded bits of each stream onto the labels of an expanded constellation according to a time-varying pattern designed for providing the same level of protection against errors to all streams. Using this scheme, multiple data streams can be concurrently transmitted on the same time-frequency resource blocks of OFDMA systems, thus achieving a higher aggregate spectral efficiency and tighter delay constraints.
\end{abstract}

\section{INTRODUCTION}

In many ways, wireless connectivity in the next decade will be conceptually different than today. Among ways foreseen by the research community are, for example, pervasive connection of machine devices, widespread (industrial) applications where wirelessly connected sensors and actuators play an important role, and, in general, an increasingly efficient and flexible use of the spectrum resources by a massive number of devices.

In this paper we focus on the need for a future network to simultaneously serve a huge number of users with high reliability and under stringent delay requirements. Aggregate data rates are likely to increase, either in the form of an increased number of devices connected to a single network access node, or in the form of multiple data streams concurrently transmitted to the same device (for example, where each stream corresponds to a different application running on the same device).

The physical layer design of today's cellular systems, including 3GPP's latest LTE system, are structurally limited in their support of these emerging targets and requirements. In particular, the orthogonal time-frequency multiple-access (MA) paradigm that reigns the LTE system today, allows only a very limited number of concurrent users simultaneous access to the spectrum resources. Given the fixed and limited number of separate frequency resources in LTE, a large number of served users can only be accommodated through an additional orthogonal time-division MA (beyond the natural orthogonal frequency-division MA) which, however, necessarily comes

The work of Jaap van de Beek was carried out for Huawei Technologies Sweden AB. with a larger latency for each user. Regimes with tight delay requirements and huge number of devices are therefore problematic.

In this paper we approach this engineering challenge by overloading the physical time-frequency resources in the LTE system. We contribute to the body of work where the concept of OverLoaded Multiple Access (OLMA) is applied to the downlink of a cellular system and where UEs with similar physical communication channel qualities are served simultaneously as, for example, in Low-Density Spread (LDS) Multiple Access (LDSMA) [1]-[3], Enhanced Trellis-Coded Multiple Access (ETCMA) [4] and Turbo Trellis-Coded Multiple Access (TTCMA) [5]. In this regime, UEs that report a similar channel quality indicator (CQI) value are grouped by a central resource scheduler and served simultaneously using the same set of physical resources. The corresponding OLMA schemes preserve the same data rate, the same transmitted energy per bit of each multiplexed stream, and the same scheduler design as if each of the multiplexed streams would have been transmitted alone on the observed time-frequencyspace resources. Furthermore, the transmit power per RE is increased proportionally to the overloading factor (the number of multiplexed streams).

The schemes in [1]-[5] perform stream multiplexing by superposition of modulated signals. As a result, the transmitted signal symbols typically belong to a constellation that is different from conventional, well-studied and practically used modulation constellations, such as QPSK and $M$-QAM. Such unconventional constellations may cause difficulties in the design of RF chains in both the transmitter and corresponding receivers. To avoid these shortcomings, we propose a new OLMA scheme called Constellation Expansion Multiple Access (CEMA), which enables the use of conventional modulation schemes available in any standard communication device. Along with the above-mentioned advantage of RF design, this scheme offers a backwards compatibility in that any conventional bit-interleaved coding scheme can be adapted to CEMA with very little effort.

Stream multiplexing over modulation symbols has been previously proposed in the context of broadcast transmission, e.g., in the form of hierarchical modulation [6] or bit-division multiplexing [7]. However, such solutions cannot be consid- 


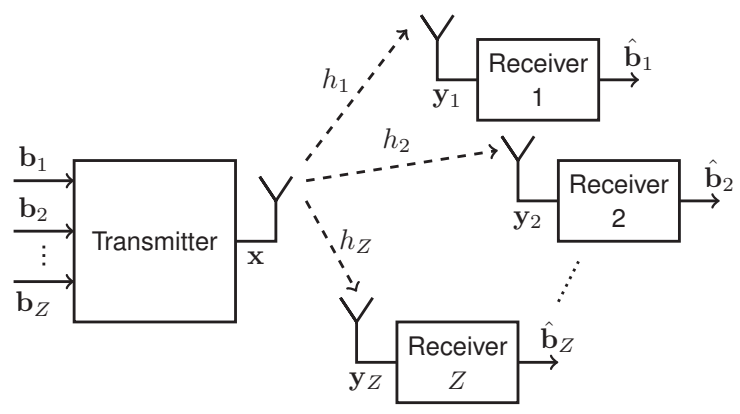

Fig. 1. System model.

ered as OLMA because their modulation format is fixed in advance depending on some system targets as, for example, the coverage area.

The paper is organized as follows: in Sec. II the CEMA concept is presented. In Sec. III we evaluate the performance of the newly proposed scheme and Sec. IV draws the final conclusions.

\section{CEMA CONCEPT}

CEMA addresses the problem of transmission to users experiencing similar SNRs and aims at providing to these users the same data rates and error protection levels as illustrated in Fig. 1. The transmitter wishes to serve multiple users simultaneously by concurrently transmitting several data streams with the maximum possible data rates. $Z$ information words $\mathbf{b}_{1}, \ldots, \mathbf{b}_{Z}$, each belonging to a different stream, are sent at the transmitter input in each transmission interval. These words (or a subset thereof) are encoded and multiplexed onto the transmitted signal $\mathrm{x}$ which consists of $L$ complex symbols. The channel from the transmitter to receiver $z \in\{1, \ldots, Z\}$ is represented by a complex coefficient $h_{z}$ modeling the combined effect of propagation path loss, shadow fading and fast fading. The received signal is

$$
\mathbf{y}_{z}=h_{z} \mathbf{x}+\mathbf{w}_{z}
$$

where $\mathbf{w}_{z}$ is a vector representing additive white Gaussian noise (AWGN) whose elements are circularly symmetric, zero-mean iid Gaussian random variables with variance $\sigma_{\mathrm{W}}=N_{0} / 2$. Here, $N_{0}$ is the two-sided power spectral density of noise. We assume that all the receivers are characterized by the same noise figure and operate at the same temperature.

A detailed scheme of CEMA transmitter is shown in Fig. 2. The CQI related to all $Z$ users are obtained through a reverse link or through local link quality assessment (e.g., in case of TDD transmission) and a subset of $U$ users characterized by the same CQI are selected for concurrent transmission. The selected users are therefore characterized by

$$
\left|h_{1}\right| \simeq\left|h_{2}\right| \simeq \ldots \simeq\left|h_{U}\right| .
$$

The CEMA transmitter performs concurrent transmission of at least one data streams to each of the selected users. Each data stream consists of a sequence of information words $\mathbf{b}_{u}=$ $\left(b_{u, 0}, \ldots, b_{u, K_{u}-1}\right)$, where $u \in\{1, \ldots, U\}$. Hereinafter, we will consider a system transmitting exactly one stream to each user. Moreover, we will assume that receivers have perfect channel state information.

When embedded in a real system, the CEMA scheduler operates in a similar way as a conventional LTE system scheduler: in each transmission interval, the CEMA scheduler computes the number $L_{z}$ of REs that will be allocated to user $z$ in the current transmission interval. Moreover, it computes a code rate $R_{z}$ and a modulation order $m_{z}$ for each user $z \in\{1, \ldots, Z\}$. Then, a subset consisting of $U$ users characterized by the same CQI is selected. The strategy employed by the CEMA scheduler to select such subset will not be discussed hereinafter, since it is out of the scope of this paper. Since all users have the same CQI, we assume

$$
\begin{aligned}
m_{1} & =\ldots=m_{U}=m_{0} \\
R_{1} & =\ldots=R_{U}=R_{0} \\
L_{1} & =\ldots=L_{U}=L .
\end{aligned}
$$

After user aggregation, the CEMA scheduler computes the order of the expanded constellation $\chi_{\mathrm{EXP}}$ that will be used for transmission as

$$
m=\log _{2}\left|\chi_{\mathrm{EXP}}\right|=U m_{0} .
$$

Information words $\mathbf{b}_{u}$ are independently encoded, then ratematching is applied, obtaining codewords $\mathbf{c}_{u}$ of length $E$ bits. These coded bits are mapped onto $m$-bit labels and a vector $\mathbf{l}=\left(l_{0}, \ldots, l_{L-1}\right)$ is formed. Each element of $\mathbf{l}$ is used by the modulator to select a corresponding symbols from $\chi_{\mathrm{EXP}}$, forming a vector $\mathrm{x}$ of complex symbols transmitted in $L$ REs.

We assume that $\chi_{\mathrm{EXP}}$ is a unit-energy constellation, therefore the SNR experienced by user $z$ is $\eta_{z}=\left|h_{z}\right|^{2} / N_{0}$. It follows form (2) that all users experience similar SNR.

\section{A. Constellations and their Bit-Level Capacities}

The concept of bit-level capacity of constellations has been defined in [8]. As discussed therein, each bit in the binary label of any constellation conveys a certain amount of information which depends on the SNR, on the particular shape of the constellation and on its specific labeling.

Fig. 3 shows the bit-level capacities of the 16QAM constellation and of the 16PSK constellation with Gray labeling. The bit-level capacities are shown as a function of the symbol energy to noise ratio $E_{s} / N_{0}$.

From Fig. 3(a), we observe that label bits $a_{1}$ and $a_{2}$ exhibit the same bit-level capacity for all SNR values. Similarly, label bits $a_{3}$ and $a_{4}$ exhibit the same bit-level capacity. However, their capacity is lower than the capacity of bits $a_{1}$ and $a_{2}$ for all SNR values. We will call bits $a_{1}$ and $a_{2}$ highly protected or strong bits, since they enjoy better error protection thanks to their larger capacity. Bits $a_{3}$ and $a_{4}$ will be called less protected or weak bits.

Fig. 3(b) shows the bit-level capacities of 16PSK. Here, label bits are characterized by three different capacities: a high capacity, an intermediate (mid) capacity and a low capacity level. These intrinsic characteristics of modulations have to 
be taken into account it the design of the coded bit-to-label bit mapping in order to provide equal levels of error protection.

\section{B. Design of the Dynamic Stream-to-Label Mapper}

In order to obtain equal protection levels for all streams and for all combinations of parameters $m_{0}$ and $U$, suitable labeling approaches have to be devised. The dynamic streamto-label mapping block of Fig. 2 associates the $m_{0}$ coded bits of each stream to the $m$ bits of the symbol label according to a mapping that changes from symbol to symbol with a predefined period of $Q$ symbol intervals. Typically, we have $Q \ll L$. Tab. I(a) shows a dynamic mapping scheme of period $Q=2$ symbols for a CEMA scheme using 16PSK as its expanded constellation. According to Tab. I(a), in each symbol interval all streams enjoy the high protection level provided by a strong bit and a lower protection level from a weaker bit . By alternately associating mid-protected bit and weak bit to the two streams, the average level of protection of the two streams becomes equal.

TABLE I

DYNAMIC STREAM TO LABEL MAPPING OF TWO STREAMS

(a) $16 \mathrm{PSK}$.

\begin{tabular}{|c|c|c|}
\hline $\begin{array}{c}\text { Dynamic } \\
\text { mapping }\end{array}$ & $\begin{array}{c}\text { First symbol } \\
\text { interval }\end{array}$ & $\begin{array}{c}\text { Second symbol } \\
\text { interval }\end{array}$ \\
\hline \multirow{2}{*}{ Stream 1 } & Strong $\left(a_{1}\right)$ & Strong $\left(a_{2}\right)$ \\
\cline { 2 - 3 } & $\operatorname{Mid}\left(a_{3}\right)$ & Weak $\left(a_{4}\right)$ \\
\hline \multirow{2}{*}{ Stream 2 } & Strong $\left(a_{2}\right)$ & Strong $\left(a_{1}\right)$ \\
\cline { 2 - 3 } & Weak $\left(a_{4}\right)$ & Mid $\left(a_{3}\right)$ \\
\hline
\end{tabular}

(b) 16QAM

\begin{tabular}{|c|c|c|}
\hline $\begin{array}{c}\text { Dynamic } \\
\text { mapping }\end{array}$ & $\begin{array}{c}\text { First symbol } \\
\text { interval }\end{array}$ & $\begin{array}{c}\text { Second symbol } \\
\text { interval }\end{array}$ \\
\hline \multirow{2}{*}{ Stream 1 } & Strong $\left(a_{1}\right)$ & Strong $\left(a_{2}\right)$ \\
\cline { 2 - 3 } & Weak $\left(a_{3}\right)$ & Weak $\left(a_{4}\right)$ \\
\hline \multirow{2}{*}{ Stream 2 } & Strong $\left(a_{2}\right)$ & Strong $\left(a_{1}\right)$ \\
\cline { 2 - 3 } & Weak $\left(a_{4}\right)$ & Weak $\left(a_{3}\right)$ \\
\hline
\end{tabular}

Using the same approach as with 16PSK, the mapping of Tab. I(b) has been designed for the case of a 16QAM expanded constellation. In QAM constellations, we observe that certain label bit pairs exhibit the same ideal bit-level capacities for
TABLE II

GENERAL DYNAMIC STREAM-TO-LABEL MAPPING SCHEME.

\begin{tabular}{c||c|c|c|c}
\multicolumn{1}{c||}{ Label bit } & \multicolumn{4}{c}{ Symbol interval } \\
& 1 & 2 & $\ldots$ & $Q$ \\
\hline$a_{1}$ & $d_{1,1}$ & $d_{2,1}$ & $\ldots$ & $d_{U, 1}$ \\
$\ldots$ & $\ldots$ & $\ldots$ & $\ldots$ & $\ldots$ \\
$a_{m_{0}}$ & $d_{1, m_{0}}$ & $d_{2, m_{0}}$ & $\ldots$ & $d_{U, m_{0}}$ \\
\hline$\ldots$ & $\ldots$ & $\ldots$ & $\ldots$ & $\ldots$ \\
\hline$a_{(U-1) m_{0}+1}$ & $d_{U, 1}$ & $d_{1,1}$ & $\ldots$ & $d_{U-1,1}$ \\
$\ldots$ & $\ldots$ & $\ldots$ & $\ldots$ & $\ldots$ \\
$a_{m}$ & $d_{U, m_{0}}$ & $d_{1, m_{0}}$ & $\ldots$ & $d_{U-1, m_{0}}$
\end{tabular}

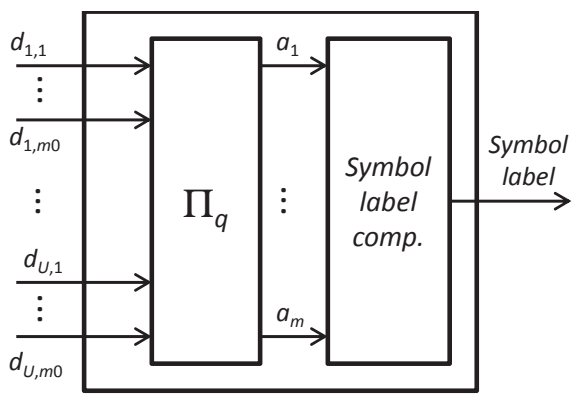

Fig. 4. Dynamic stream-to-label mapper.

each given SNR value. In practice, however, these pairs might be characterized by different levels due to impairments caused by the transceivers' analog RF/IF sections, therefore we treat each bit in the label as being characterized by distinct capacity levels.

In general, the number of distinct bit-level capacities is at most equal to the constellation order $m$. A dynamic mapping scheme that provides equal error protection to all streams even in such case is shown in Tab. II. A key property of this scheme is that each label bit $a_{i}$ is cyclically assigned to all streams according to a periodic pattern. The resulting mapping achieves equal protection levels on all streams for all values of $U$ and $m$ with a mapping period of $Q=U$ symbols.

Fig. 4 shows a block scheme in which the dynamic streamto-label mapper is represented as the cascade of a time-varying interleaver which operates according to a set of permutations

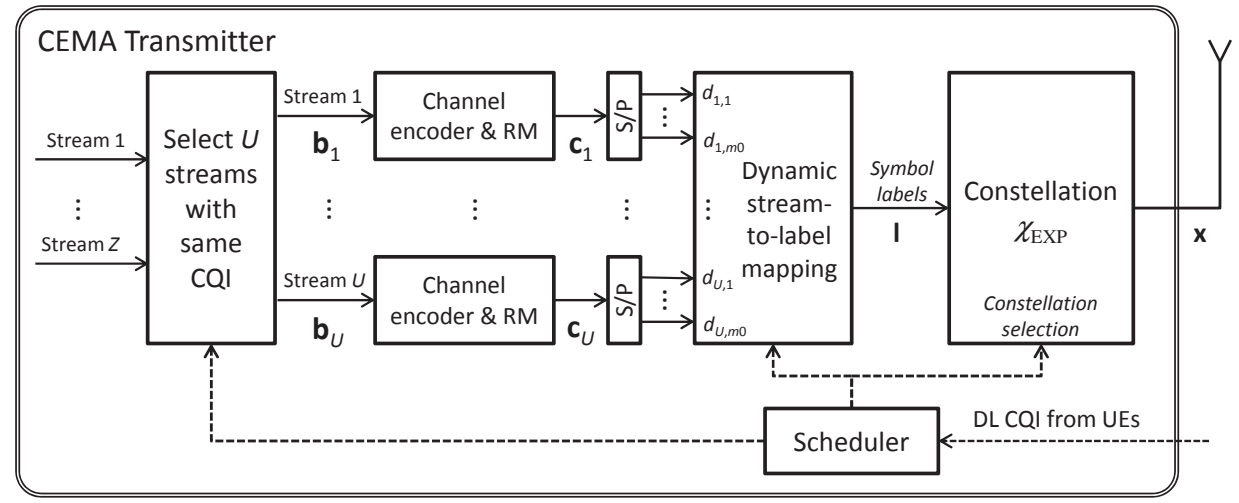

Fig. 2. CEMA transmitter. Solid arrows indicate data signals. Dashed arrows indicate control signals. 


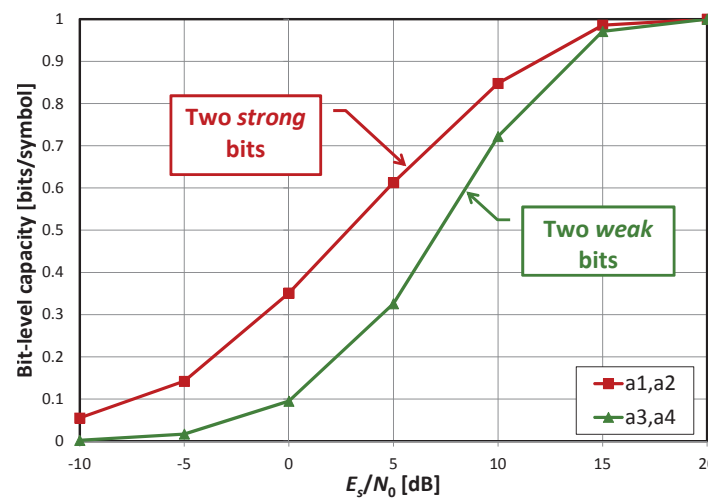

(a) Bit-level capacities of 16QAM with Gray labeling.

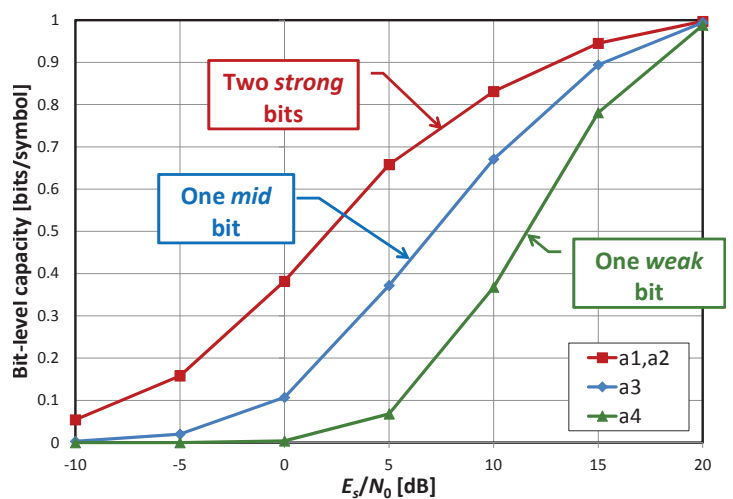

(b) Bit-level capacities of 16PSK with Gray labeling.

Fig. 3. Bit-level capacities of 16QAM and 16PSK constellations with Gray labeling.

$\left(\Pi_{q}\right)_{q=1}^{Q}$ and a block that computes the symbol label

$$
l=\sum_{i=1}^{m} a_{i} 2^{i-1}
$$

that will be used by the modulator to select the transmitted symbol from $\chi_{\mathrm{EXP}}$.

\section{CEMA Receiver}

The receiver performs detection and decoding as shown in the simulation scheme of Fig. 5, delivering an estimate $\hat{\mathbf{b}}_{u}$ of the information word $\mathbf{b}_{u}$ to the corresponding recipient. We assume that perfect knowledge of channel coefficients $h_{u}$ is available.

The detector computes the log-likelihood ratios (LLRs) of symbols $s_{n}$ of the expanded constellation $\chi_{\mathrm{EXP}}$ as

$$
\kappa_{n, k}^{(u)}=\log \frac{P\left(x_{k}=s_{n} \mid y_{u, k}\right)}{P\left(x_{k}=s_{0} \mid y_{u, k}\right)}, n=0, \ldots,\left|\chi_{\mathrm{EXP}}\right|-1
$$

where $k \in\{0, \ldots, L-1\}$ is the time index, $x_{k}$ is the symbol transmitted at time $k, s_{n} \in \chi_{\mathrm{EXP}}$ is a symbol of the expanded constellation and $y_{u, k}$ is the complex sample received by user $u$ at time $k$. After computing $\kappa_{n, k}^{(u)}$, the detector computes the binary LLRs of codeword $\mathbf{c}_{u}=\left(c_{u, 0}, \ldots, c_{u, E-1}\right)$ as

$$
\begin{aligned}
\lambda_{u, j} & =\log \frac{P\left(c_{u, j}=1 \mid \mathbf{y}_{u}\right)}{P\left(c_{u, j}=0 \mid \mathbf{y}_{u}\right)} \\
& =\log \sum_{n: \mathcal{L}_{\xi_{u}(j)}\left(s_{n}\right)=1} \exp \left(\kappa_{n, \omega_{u}(j)}^{(u)}\right) \\
& -\log \sum_{n: \mathcal{L}_{\xi_{u}(j)}\left(s_{n}\right)=0} \exp \left(\kappa_{n, \omega_{u}(j)}^{(u)}\right)
\end{aligned}
$$

where $\omega_{u}(j) \in\{0, \ldots, L-1\}$ indicates the symbol in which bit $j$ of $\mathbf{c}_{u}$ has been transmitted and $\xi_{u}(j) \in\{0, \ldots, m-1\}$ indicates its position in the binary label. Here, $\mathcal{L}_{\xi}\left(s_{n}\right)$ indicates the value of bit $\xi$ in the binary label associated to constellation symbol $s_{n}$.

The computed LLRs are sent to the iterative turbo decoder which computes the a-posteriori LLRs of information bits by iteratively executing the soft-in soft-out (SISO) algorithm [9]. Finally, an estimate $\hat{\mathbf{b}}_{u}$ of information word $\mathbf{b}_{u}$ is computed and delivered to the recipient.

An improved receiver scheme performing iterative detection and decoding as in [10] has also been implemented. At each receiver iteration, the turbo decoder performs a given number of decoding iterations, after which it computes $a$ posteriori extrinsic LLRs of coded bits. These LLRs are fed back to the detector and used as a-priori information on the received constellation symbols. The LLRs of symbols $s_{n}$ are recomputed as in (4) taking into account the new $a$ priori information. Symbol LLRs $\kappa_{n, k}^{(u)}$ are updated after each decoder execution, as in Succesive Interference Cancellation (SIC) schemes [11]. During the last receiver iteration, the turbo decoder computes the a-posteriori LLRs of information bits that are used to obtain the decoded information word $\hat{\mathbf{b}}_{u}$.

\section{RESULTS}

We evaluate by Monte Carlo simulation the BLock Error Rate (BLER) of the considered system for several SNR values and compute the aggregate spectral efficiency as

$$
\mathrm{SE}(U, \mathrm{SNR})=(1-\mathrm{BLER}) R_{0} m_{0} U[\mathrm{bits} / \mathrm{s} / \mathrm{Hz}] .
$$

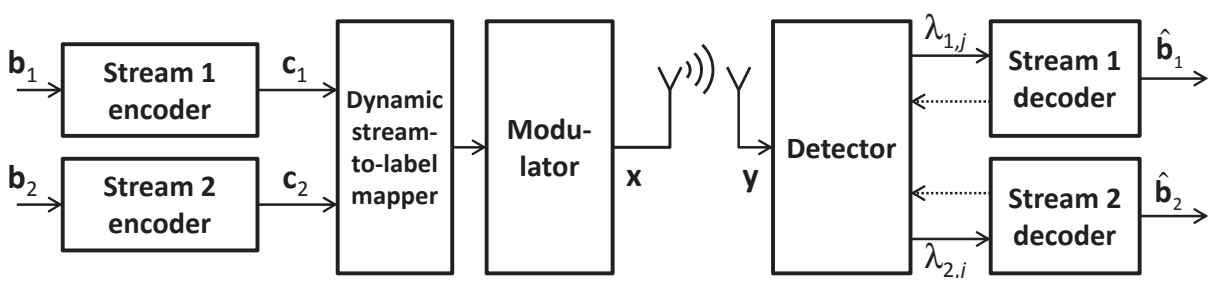

Fig. 5. CEMA scheme used for performance evaluation. Signals corresponding to dashed lines are active only in the iterative receiver scheme. 


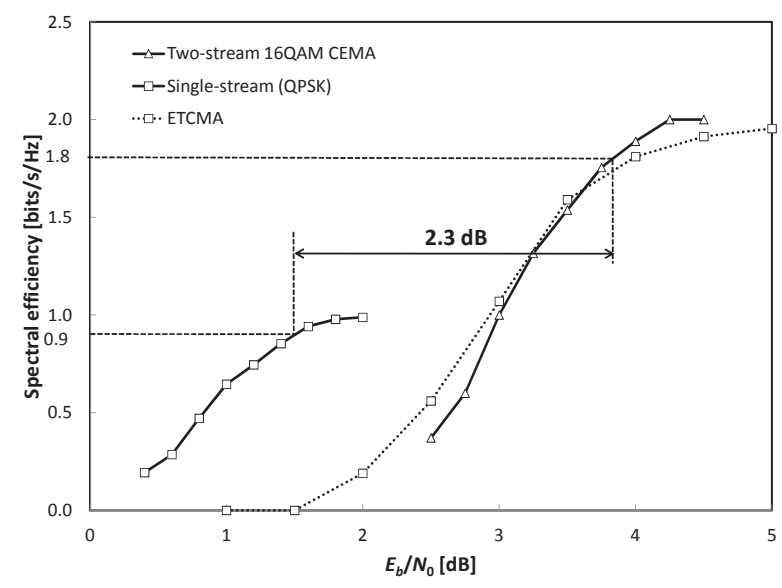

(a) Spectral efficiency of a two-user CEMA scheme using 16QAM.

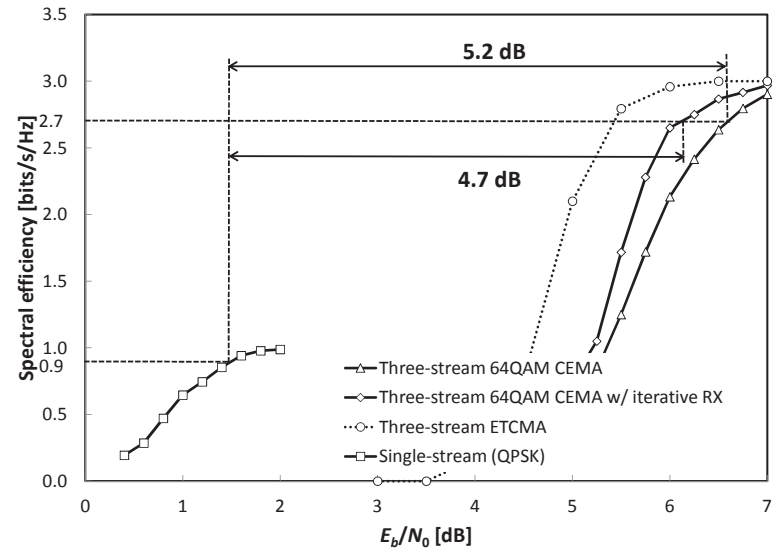

(b) Spectral efficiency of a three-user CEMA scheme using 64QAM.

Fig. 6. Spectral efficiency of two-stream and three-stream CEMA schemes compared with the SE of the single-link coded QPSK transmission.

Here, $R_{0}$ is the channel code rate, $m_{0}$ is the base modulation order in bits per symbol and $U$ is the number of multiplexed streams. We indicate with

$$
S E_{\infty}(U)=\lim _{\mathrm{SNR} \rightarrow \infty} \mathrm{SE}(U, \mathrm{SNR})
$$

the asymptotic aggregate spectral efficiency (ASE).

For each $U$, we define the operating threshold SNRs as the minimum SNR needed to achieve a certain ratio $\rho$ of the asymptotic aggregate SE:

$$
\operatorname{SNR}^{*}(U, \rho)=\min \left\{\operatorname{SNR}: \operatorname{SE}(\operatorname{SNR}, U) \geq \rho \operatorname{SE}_{\infty}(U)\right\} .
$$

The increased aggregate SE of any OLMA scheme is achieved at the expense of an increased transmitted power for each multiplexed stream/user. This increase can be characterized by the single-stream $\operatorname{SNR}$ loss $\Delta_{\mathrm{SNR}}(U, \rho)$, defined as

$$
\Delta_{\mathrm{SNR}}(U, \rho)=\operatorname{SNR}^{*}(U, \rho)-\operatorname{SNR}^{*}(1, \rho) .
$$

The CEMA scheme of Fig. 5 has been used for performance evaluation. Two data streams using QPSK as the base constellation and a code rate $R_{0}=1 / 2$ are multiplexed and concurrently transmitted. Each transmission interval consists of $L=240$ symbols. As for channel coding, the turbo code and corresponding rate-matching algorithm defined in the LTE standard [12] has been employed.

Fig. 6 shows the spectral efficiency achieved by the CEMA system on the AWGN channel as a function of the information bit energy to noise ratio, defined as

$$
\frac{E_{b}}{N_{0}}=\frac{E_{s}}{U m R_{0} N_{0}}
$$

where $E_{s}=\mathbb{E}_{n}\left[s_{n}\right]$ is the average symbol energy of $\chi_{\mathrm{EXP}}=\left\{s_{n}, n=1, \ldots, M\right\}$ and $m$ is its order. We have implicitly assumed that $\left|h_{u}\right|=1, \forall u$, i.e., all streams are characterized by the same SNR. Here, $N_{0}$ is the one-sided power spectral density of the additive white Gaussian noise. In our simulations, AWGN is the only considered channel impairment. More realistic models of wireless channels encompassing fading and interference will be considered in future works.

Fig. 6(a) shows the SE of a CEMA scheme with two streams using 16QAM as the expanded constellation. The SE of CEMA is compared with the SE of a single-user coded QPSK transmission with the same code rate and same block length. The turbo decoder executes $N_{\text {IT }}=8$ iterations; the receiver does not perform iterative detection and decoding as we observed that this does not result in improved performance in this case. The increased SE of the two-stream CEMA scheme is achieved at a higher SNR corresponding to a singlestream SNR loss $\Delta_{\mathrm{SNR}}(2,0.9)=2.3 \mathrm{~dB}$. The aggregate $\mathrm{SE}$ of a two-stream ETCMA system is also shown in Fig. 6(a). We observe that the two-stream CEMA scheme and the ETCMA scheme feature almost the same performance, with CEMA performing slightly better than ETCMA at high SNR.

Fig. 6(b) shows the SE of a CEMA scheme with three streams using 64QAM as the expanded constellation. Here, the CEMA scheme achieves a SE three times larger than the SE of the single-stream transmission with a SNR loss $\Delta_{\mathrm{SNR}}(3,0.9)=5.2 \mathrm{~dB}$. The turbo decoder executes $N_{\mathrm{IT}}=8$ iterations.

Employing a receiver that performs iterative detection and decoding, the single-stream SNR loss is reduced to $\Delta_{\mathrm{SNR}}(3,0.9)=4.7 \mathrm{~dB}$, obtaining a gain of $0.5 \mathrm{~dB}$ with respect to the non-iterative receiver. When performing iterative detection and decoding, the turbo decoder executes $N_{\text {IT, } 1}=5$ iterations during the first receiver iteration and $N_{\mathrm{IT}, 2}=7$ iterations during the second. These values have been obtained through an optimization procedure aimed at maximizing the spectral efficiency.

The aggregate SE of a three-stream ETCMA system is also shown in Fig. 6(a). We observe that the three-stream CEMA with iterative receiver exhibits a slightly worse performance with a SNR gap of about $0.7 \mathrm{~dB}$. The superior performance of ETCMA is likely due to the characteristics of its superposed constellation, while the 64-QAM constellation used by CEMA 
exhibits a shaping loss w.r.t. the AWGN channel capacity. Such shaping loss is less pronounced for 16-QAM, hence the smaller gap between the two-stream CEMA using 16-QAM and the two-stream ETCMA scheme.

The complexity increase implied by the adoption of CEMA is due to the iterations involving the detector executed when the iterative receiver is employed. However, the number of such iterations is rather low (two in our simulations), since we observed that further iterating does not result in noticeable performance improvements. As a result, the total number of turbo decoding iterations is only slightly increased.

\section{CONCLUSIONS}

We have proposed a new overloaded multiple access scheme that significantly increases the number of data streams that can be multiplexed and concurrently transmitted on the same timefrequency resources of wireless systems.

CEMA overcomes the limitations imposed by most OFDMA systems by allowing multiple data streams to be transmitted in each time-frequency resource block. This way, a higher number of streams can be concurrently transmitted, achieving a higher aggregate spectral efficiency than conventional systems while fulfilling tighter delay requirements when a large number of devices are simultaneously served.

Since CEMA uses the same conventional modulation and coding schemes available in most communication devices, its adoption in current wireless systems based on OFDMA like LTE and LTE-Advanced is made easier.

\section{REFERENCES}

[1] J. Choi, "Low density spreading for multicarrier systems," in IEEE 8th Int. Symp. Spread Spectrum Techniques and Applications, 2004, Aug 2004, pp. 575-578.

[2] J. van de Beek and B. M. Popović, "Multiple access with low-density signatures," in IEEE Global Telecommun. Conf., GLOBECOM 2009, Nov 2009, pp. 1-6.

[3] B. M. Popović, A. R. Safavi, and A. G. Perotti, "Bit-interleaved low density spread (BI-LDS) transmission," in IEEE Wireless Commun. Networking Conf., WCNC 2014, Apr 2014.

[4] A. G. Perotti and B. M. Popović, "Enhanced trellis coded multiple access (ETCMA)," in 2014 IEEE Inf. Theory Workshop (ITW), Nov 2014.

[5] — "Turbo trellis coded multiple access," in 2014 IEEE Globecom Workshops (GC Wkshps), Dec 2014.

[6] European Telecommunications Standards Institute, "ETSI EN 300744 digital video broadcasting (DVB); framing structure, channel coding and modulation for digital terrestrial television," January 2009.

[7] H. Jin, K. Peng, and J. Song, "Bit division multiplexing for broadcasting," IEEE Trans. Broadcast., vol. 59, no. 3, pp. 539-547, Sept 2013.

[8] C. Stierstorfer, R. F. Fischer, and J. B. Huber, "Optimizing BICM with convolutional codes for transmission over the AWGN channel," in International Zurich Seminar on Communications, Mar 2010.

[9] S. Benedetto, D. Divsalar, G. Montorsi, and F. Pollara, "Soft-input softoutput modules for the construction and distributed iterative decoding of code networks," European Transactions on Telecommunications, vol. 9, pp. 155-172, March 1998 .

[10] X. Li and J. Ritcey, "Bit-interleaved coded modulation with iterative decoding using soft feedback," Electronics Letters, vol. 34, no. 10, pp. 942-943, May 1998.

[11] D. Tse and P. Viswanath, Fundamentals of wireless communications. Cambridge University Press, 2005.

[12] "Third Generation Partnership Project; Technical Specification Group Radio Access Network; Evolved Universal Terrestrial Radio Access (E-UTRA); Multiplexing and channel coding (Release 12)," 3GPP TS 36.212 V12.0.0, Dec 2013. 